\title{
Substantial chest-wall deformity following tissue expansion after radiotherapy
}

\author{
Ramon P. de Wildt • Stefania Tuinder • \\ René R. W. J. van der Hulst
}

Received: 6 April 2009 /Accepted: 21 September 2009/Published online: 21 October 2009

(C) Springer-Verlag 2009

\begin{abstract}
We present the case of a 54-year-old woman who underwent a two-stage breast reconstruction with a tissue expander after sustaining a lumpectomy and local radiotherapy for breast cancer. During expansion, the woman developed an abnormal concave deformity of the chest wall. Although respiratory or aesthetic consequences were expected, our patient reported only pain and was satisfied with the end result. Osteoporosis or local recurrence was excluded as predisposing factors, and radiotherapy was considered to be the causal factor in our patient. On the basis of this finding, we advise surgeons to take the risk of chest-wall deformity into consideration when planning a reconstruction with tissue expanders, especially in patients with a history of radiotherapy, and we recommend an alternative reconstructive method in this group of patients.
\end{abstract}

Keywords Tissue expansion devices · Breast · Deformity · Thoracic wall $\cdot$ Radiotherapy $\cdot$ Complications

\section{Introduction}

Breast reconstruction after breast-cancer surgery is an important part of daily practise for most plastic surgeons, because of the high prevalence of breast cancer (lifetime prevalence of $12.7 \%$ in the Netherlands) [1] and an increasing demand for reconstructions. The two-stage

R. P. de Wildt $\cdot$ S. Tuinder $(\varangle) \cdot$ R. R. W. J. van der Hulst Department of Plastic, Reconstructive and Hand Surgery, Maastricht University Medical Centre,

Maastricht, The Netherlands

e-mail: nervofaciale@yahoo.it breast reconstruction using a tissue expander, followed by a permanent implant, is a relatively simple method which yields good aesthetic results. However, several complications associated with this procedure-including hematoma, infection, and capsular contraction - have been well described in the literature. A less documented complication is that of chest-wall deformity following tissue expansion. It has been described before, but predominantly in osteoporotic women $[2,3]$ and in patients whose expanders were significantly overinflated $[4,5]$.

In this article, we describe the case of a woman who developed a chest-wall deformity following normal volume tissue expansion after radiotherapy and then provide a review of the literature on this subject.

\section{Case}

In April 2008, a 54-year old, postmenopausal, nonosteoporotic woman was referred to our outpatient clinic for a delayed breast reconstruction following a lumpectomy and sentinel lymph node procedure undergone a year earlier, for a T1N0Mx grade III carcinoma of the left breast. Subsequent to surgery, the patient had received adjuvant chemotherapy and 33 fractions of radiotherapy, amounting a total dose of $66 \mathrm{~Gy}$.

The patient had a further history of hypertension and polycystic kidney disease. She used tamoxifen and did not smoke. As a result of the lumpectomy, the left breast had a retracted lower pole (Fig. 1). The contralateral breast was relatively small (A cup).

Several reconstructive options were discussed, and our patient chose a two-stage reconstruction with a tissue expander. The higher rate of complications associated 
Fig. 1 Appearance of left breast after lumpectomy and radiotherapy, prior to placement of tissue expander
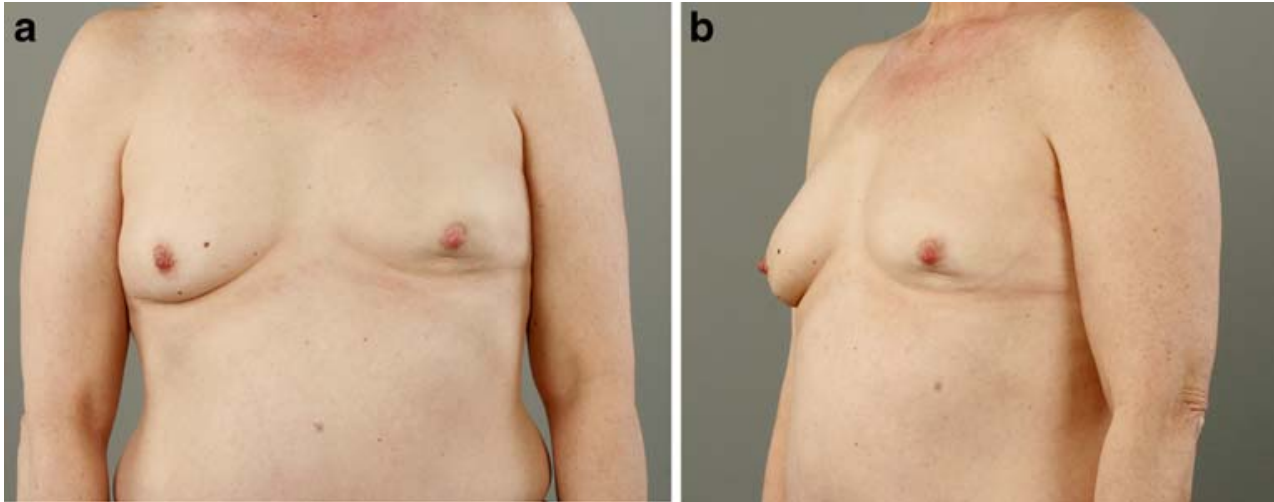

with radiotherapy, such as delayed wound healing, wound dehiscence, and capsular contraction, were discussed with the patient.

In May 2008, a 350-ml medium contour profile tissue expander (Mentor ${ }^{\circledR}$ Corp., Santa Barbara, CA, USA) was placed in the subpectoral plane and peroperatively infused with $50 \mathrm{ml}$ of saline. After the wound had healed, the volume of the saline in the tissue expander was increased at the rate of $25 \mathrm{ml}$ a week. The patient reported considerable pain during and after each inflation. After 3 months, the expander had reached a total volume of $355 \mathrm{ml}$; at that time, a second operation was performed in which the expander was removed, and a permanent 345-ml medium high profile breast implant (Mentor ${ }^{\circledR}$ Corp., Santa Barbara, CA, USA) was placed in the left breast. In addition, a 175-ml round moderate plus profile breast implant (Mentor ${ }^{\circledR}$ Corp., Santa Barbara, CA, USA) was placed in the right breast for augmentation purpose, as desired by the patient. During surgery, the surgeon noticed an abnormal concave deformity of the chest wall directly under the place where the tissue expander had been located. To exclude local recurrence, biopsies of one of the depressed ribs were taken before the permanent implant was placed.

Postoperatively a magnetic resonance imaging (MRI) of the chest was made to evaluate (intra)thoracic abnormalities. The MRI showed a substantial depression of the ribs under the left implant, but no evidence of local recurrence and no abnormalities in other areas of the chest wall and cavity (Fig. 2). Pathologic evaluation of the biopsies excluded malignancy, and a dual energy X-ray absorptiometry scan showed normal bone density, thereby excluding osteoporosis.

During the entire reconstructive process, the patient suffered no respiratory discomfort or decreased exercise tolerance. After reconstruction of the left breast and augmentation of the right breast, our patient had a bilateral B cup and was satisfied with the end result (Fig. 3). We suggested lipofilling in a later stage to improve the contour of the lower pole of the left breast.

\section{Discussion}

Since the introduction of the tissue expander for breast reconstruction by Radovan [6] in the early 1980s, it has become a reliable and satisfying method for breastreconstruction surgery. Even in the present era of autologous
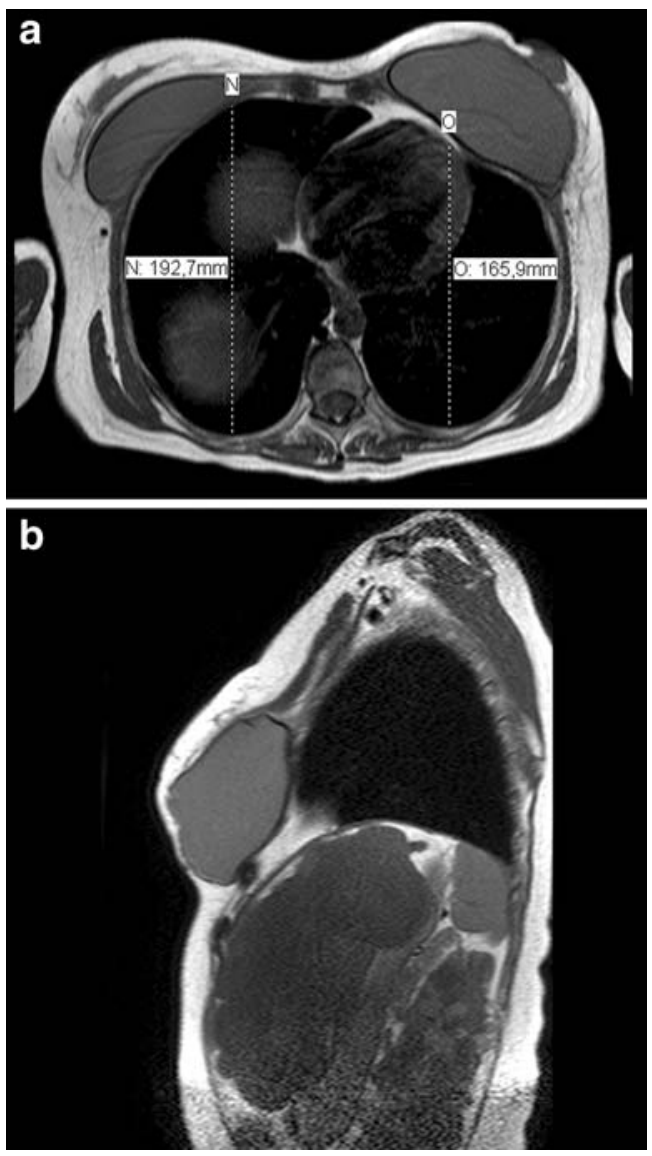

Fig. 2 Transverse (a) and sagittal (b) magnetic resonance imaging slides taken directly after placement of bilateral permanent implants. Note displacement of the ribs and decreased anteroposterior diameter of the chest wall on the left side 
Fig. 3 Appearance of left and right breast 9 months after placement of bilateral permanent implants
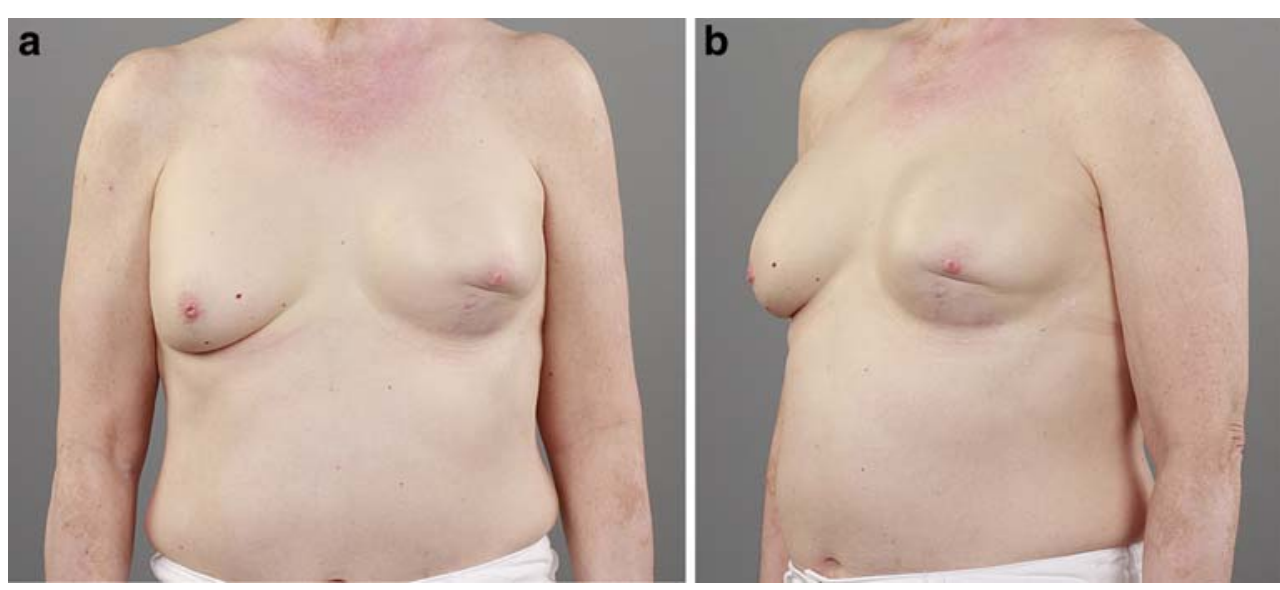

tissue reconstruction, the two-stage reconstruction is the most commonly performed method [7], mainly because of its relative simplicity, short operating time, lack of donor site morbidity, and quick patient recovery.

The phenomenon of chest-wall deformity after tissue expansion had already been described a few times in the 1980s. In 1991, Sinow et al. [5] published a prospective study on the subject, using computed tomography imaging and a semi-quantitative scale to measure the extent of deformity. They found some form of rib deformity in most patients, moderate or greater rib depression in $13 \%$ of their patients, and even an asymptomatic rib fracture in one patient. However, tissue expanders in all of their patients were overinflated to a total volume that was $40-80 \%$ greater than the size of the permanent implants the patients eventually received. One patient suffered from breathing problems and restrictive pulmonary function after a nearly 1300-ml bilateral expansion. The authors claim a positive correlation between capsular contraction and deformity, but no correlation to pain during or after expansion. Moor et al. [4] published a similar study on tissue expanders; however, they also overinflated expanders in all their patients. Fiftythree percent of patients had some chest-wall abnormality, and they also found a statistically significant increase of chest-wall deformity after delayed breast reconstruction compared to immediate reconstruction.

Minor deformation of the chest wall is frequently seen after tissue expansion $[4,5]$, but the less common moderate to severe chest-wall deformity is a more serious complication, and one should take into account that it can lead to considerable morbidity [5]. It is widely known that radiotherapy is associated with a substantially higher complication rate in prosthetic breast reconstruction [7], worsened aesthetic outcome, and significantly increased capsular contraction in immediate breast reconstruction with implants [8]. Similar to the former complications, the chance of chest-wall deformation after tissue expansion is most likely increased by irradiation. Whether this is due to decreased strength of the ribs or tightening of the overlying soft tissue is unknown [5].

The effects of irradiation on skin and soft tissue can often be observed in patients, but the effect on bone strength is something surgeons are less familiar with. Radiotherapy is associated with radiographically detected osteoporosis, and investigations have shown bone mineral density to be reduced or fragility to be increased after irradiation, but the precise pathophysiology is not known [9]. There is a significantly higher incidence of spontaneous rib fractures in postmastectomy breast-cancer patients treated with large-dose irradiation than in patients treated with standard-dose irradiation [10]. This emphasises the potential lack of rib strength following this adjuvant treatment. It becomes even more clinically relevant when an external force is applied to the chest wall, such as in the course of tissue expansion.

Various techniques can be used to reconstruct lumpectomy or partial mastectomy defects with or without a history of radiotherapy. For small defects or concavities, multistage lipofilling with autologous fat is a good option. Larger defects often require a pedicled latissimus dorsi flap with or without a breast implant or a two-stage reconstruction using a tissue expander. Furthermore, free flaps, such as a deep inferior epigastric perforator flap, can be harvested and transplanted to the breast. Finally, a patient with a tumour in the lower pole of the breast may be eligible for tumour excision in the pattern of an inverted-T or vertical-scar mammaplasty, with or without contralateral symmetrization. Obviously, this technique is not possible in patients who have already undergone lumpectomy.

In patients with a history of radiotherapy to the breast, we prefer a reconstruction with autologous tissue from a nonradiated donor site, such as a pedicled or free flap, or lipofilling. In this particular case, a pedicled latissimus dorsi flap would be our first choice for reconstruction of the lower pole.

Radiotherapy is a relative contra-indication for the use of tissue expanders in breast reconstruction. We therefore 
advise surgeons to counsel patients with a history of radiotherapy to opt for an alternative reconstructive method using autologous tissue, because of the associated increased risk of complications, including deformity of the chest wall. If, nonetheless, tissue expanders are used, one should exercise caution and be on the lookout for any signs indicating a developing chest-wall deformity.

\section{References}

1. Kiemeney LA, Lemmers FA, Verhoeven RH, Aben KK, Honing C, de Nooijer J, Peeters PH, Visser O, Vlems FA (2008) The risk of cancer in the Netherlands. Ned Tijdschr Geneeskd 152:2233-2241

2. McKinney P, Edelson R, Terrasse A, Zukowski M (1987) Chest-wall deformity following soft-tissue expansion for breast reconstruction. Plast Reconstr Surg 80:442-444

3. Sariguney Y, Ayhan S, Eryilmaz T (2008) Chest wall deformity after tissue expansion. Scand J Plast Reconstr Surg Hand Surg 42:108-109
4. Moor EV, Wexler MR, Bar-Ziv Y, Weinberg A, Chaouat M, Ad-El D, Raveh T, Alfie M, Caspi R, Neuman A (1996) Chest wall deformity following maximal tissue expansion for breast reconstruction. Ann Plast Surg 36:129-132

5. Sinow JD, Halvorsen RA Jr, Matts JP, Schubert W, Letourneau JG, Cunningham BL (1991) Chest-wall deformity after tissue expansion for breast reconstruction. Plast Reconstr Surg 88:998-1004

6. Radovan C (1982) Breast reconstruction after mastectomy using the temporary expander. Plast Reconstr Surg 69:195-208

7. Spear SL, Mesbahi AN (2007) Implant-based reconstruction. Clin Plast Surg 34:63-73

8. Behranwala KA, Dua RS, Ross GM, Ward A, A'Hern R, Gui GP (2006) The influence of radiotherapy on capsule formation and aesthetic outcome after immediate breast reconstruction using biodimensional anatomical expander implants. J Plast Reconstr Aesthet Surg 59:1043-1051

9. Hopewell JW (2003) Radiation-therapy effects on bone density. Med Pediatr Oncol 41:208-211

10. Overgaard M (1988) Spontaneous radiation-induced rib fractures in breast cancer patients treated with postmastectomy irradiation. A clinical radiobiological analysis of the influence of fraction size and dose-response relationships on late bone damage. Acta Oncol $27: 117-122$ 